\title{
Exciton effects in a scaling theory of intermediate valence and Kondo systems
}

\author{
V. Yu. Irkhin and M. I. Katsnelson \\ Institute of Metal Physics, Ekaterinburg 620219, Russia \\ Department of Physics, Uppsala University, Uppsala 75121 Sweden
}

\begin{abstract}
An interplay of the Kondo scattering and exciton effects ( $d-f$ Coulomb interaction) in the intermediate valence systems and Kondo lattices is demonstrated to lead to an essential change of the scaling behavior in comparison with the standard Anderson model. In particular, a marginal regime can occur where characteristic fluctuation rate is proportional to flow cutoff parameter. In this regime the "Kondo temperature" itself is strongly temperature dependent which may give a key to the interpretation of controversial experimental data for heavy fermion and related systems.
\end{abstract}

PACS numbers: 71.27.+a, 71.28.+d, 75.30.Mb

There is an interesting class of rare-earth compounds such as $\mathrm{Ce}_{4} \mathrm{Bi}_{3} \mathrm{Pt}_{3}, \mathrm{SmB}_{6}, \mathrm{SmS}$ under pressure (the "golden" phase), TmSe, $\mathrm{YbB}_{12}$ which were called earlier intermediate valence (IV) compounds and now are treated as "heavy-fermion (HF) semiconductors", or "Kondo insulators" (for a review, see Refs 1, 2). Various names emphasize different peculiarities of these compounds. As for electron energy spectrum, most of them are narrow gap semiconductors with an anomalously small energy scale (the gap width), of the order of tens or hundreds of Kelvins (see Ref 2 and a review of earlier experiments in Ref. (3)). At the same time, they do demonstrate intermediate valence of the rare earth ions (usually between $2+$ and $3+$ ) in a number of properties, e.g., lattice constants (which are intermediate between those for isostructural compounds with di- and trivalent ions), core level spectra (which are a mixture of the spectra of di- and trivalent ions with comparable weights), and many others $[1,4,5]$.

As well as for the HF metals, the origin of this small energy scale is a key point to understand anomalous properties of the IV compounds. For the HF metals it is commonly accepted now that they are the Kondo lattices, which means that this energy scale (the Kondo temperature $T_{K}$ ) is a width of the Kondo resonance owing to spin-dependent scattering of conduction electrons by $f$ electron centers 6]. As a result of an interplay of the Kondo effect and interatomic magnetic interactions, the $T_{K}$ value for a lattice can strongly differ from that for an isolated impurity 7], spin fluctuations being of crucial importance for the HF behavior. It is very natural to expect that these effects are important also for the IV compounds. At the same time, valence or charge fluctuations should be also considered. They are determined in part by the Coulomb ("Falicov-Kimball") interaction between conduction and localized electrons [8]. Taking into account these interactions together with the hybridization processes it is possible to describe the IV state as a kind of exciton condensation 3, 9]. Note that in the IV regime the spinless Falicov-Kimball model with hybridization is formally equivalent to the anisotropic Kondo problem, different valent states playing the role of pseudospin "up" and "down" states [10]. It is the degeneracy of quantum states for a scattering center which is important for the formation of the Kondo resonance 11. In the IV case the divalent and trivalent states are degenerate by definition, so that this analogy is not surprising. Therefore it is natural to consider the formation of the Kondo resonance for the IV compounds taking into account both spin and charge fluctuations, or, equvalently, both the "Kondo" and exciton ("Falicov-Kimball") effects. This is the aim of the present work. Since there is no clear demarcation between the IV and Kondo systems, it will be shown that the excitonic effects may be relevant also for the latter case.

To investigate effects of interaction of current carriers with local moments we use the Hamiltonian of the asymmetric infinite- $U S U(N)$ Anderson model with inclusion of the Falicov-Kimball interaction (on-site $d-f$ Coulomb repulsion $G$ ),

$$
\begin{aligned}
\mathcal{H}= & \sum_{\mathbf{k} m}\left[t_{\mathbf{k}} c_{\mathbf{k} m}^{\dagger} c_{\mathbf{k} m}+V\left(c_{\mathbf{k} m}^{\dagger} f_{\mathbf{k} m}+f_{\mathbf{k} m}^{\dagger} c_{\mathbf{k} m}\right)\right. \\
& \left.+E_{f} f_{\mathbf{k} m}^{\dagger} f_{\mathbf{k} m}\right]+G \sum_{i m m^{\prime}} f_{i m}^{\dagger} f_{i m} c_{i m^{\prime}}^{\dagger} c_{i m^{\prime}}
\end{aligned}
$$

where the on-site $f-f$ Coulomb interaction is put to infinity, so that doubly occupied states are suppressed, $f_{i m}^{\dagger}=|i m\rangle\langle i 0|$ are the Hubbard operator $(|i m\rangle$ and $|i 0\rangle$ are single-occupied and empty states), we neglect for simplicity the k-dependence of the hybridization $V$. Note that similar calculations can be performed for realistic rare-earth ions, including the case of two magnetic configurations, see Ref. 12.

Following to Ref. 10, we treat the coherent and incoherent cases. In the first case dispersion in the spectrum of $f$-electrons occurs. For simplicity this is supposed to be proportional to the conduction electron spectrum, $E_{f \mathbf{k}}=E_{f}+\lambda \varepsilon_{\mathbf{k}}, \varepsilon_{\mathbf{k}} \propto t_{\mathbf{k}}, \lambda=-1$ (the $f$-band has a hole character). In the incoherent regime $\lambda=0$, so that the $f$-electrons remain localized. Note that in the presence of the energy gap we always deal with the coherent regime.

The renormalization of the Coulomb parameter $G$ and the hybridization $V$ is obtained, similar to Ref. 10, from the two-particle Green's function

$$
F_{m m^{\prime}}^{\sigma}(E)=\left\langle\left\langle f_{i m}^{\dagger} c_{i m^{\prime}} \mid c_{i m^{\prime}}^{\dagger} f_{i m}\right\rangle\right\rangle_{E}
$$


which determines the vertex. We obtain the singular correction with the structure

$$
\delta F_{m m^{\prime}}^{\sigma}(E)=G F_{m m^{\prime}}^{\sigma}(E) \sum_{\mathbf{q}} \frac{n_{\mathbf{q} m^{\prime}}}{E-t_{\mathbf{q}}+E_{f}}
$$

where $n_{\mathbf{k} m}=\left\langle c_{\mathbf{k} m}^{\dagger} c_{\mathbf{k} m}\right\rangle$ is the Fermi function. In the coherent regime, a similar correction occurs from the dispersion of $f$-states. The correction (3) contains a logarithmic Kondo-like divergence owing to charge fluctuations, which is cut at $E_{f}$ (the latter quantity plays the role of the external field in the equivalent anisotropic Kondo model). Unlike the renormalization of $E_{f}$, the renormalizations of $V$ and $G$ do not contain the degeneracy factor of $N$.

The renormalization of $E_{f}$ owing to spin-flip processes is obtained in the second order in hybridization (cf. 12, 13, 18])

$$
\delta E_{f m}=V^{2} \sum_{m^{\prime} \neq m, \mathbf{q}} \frac{n_{\mathbf{q} m^{\prime}}}{E_{F}-t_{\mathbf{q}}}+n \delta G
$$

We have taken into account in Eq. (44) the Hartree renormalization of $f$-level energy, which occurs in the coherent case, $E_{f} \rightarrow E_{f}+G n, n$ being the concentration of conduction electrons; we put in numerical calculations $n=1$, which corresponds to the Kondo regime.

To derive the scaling equations for the effective model parameters we use the poor-man scaling approach 14]. Picking out in the integrals with the Fermi functions (3), (44) the contributions from the energy layer $C<E<$ $C+\delta C$ near the Fermi level $E_{F}=0$ and replacing $E_{f} \rightarrow$ $E_{f}(C), V \rightarrow V(C), G \rightarrow G(C)$ we obtain (cf. Ref. 10)

$$
\begin{aligned}
\frac{\partial E_{f}(C)}{\partial \ln C} & =-\rho(N-1) V^{2}(C)+n \frac{\partial G(C)}{\partial \ln |C|} \\
\frac{\partial V(C)}{\partial \ln \left|C-E_{f}\right|} & =-\frac{1-\lambda}{1+|\lambda|} \rho V(C) G(C) \\
\frac{\partial G(C)}{\partial \ln \left|C-E_{f}\right|} & =\frac{2 \lambda}{1+|\lambda|} \rho G^{2}(C)
\end{aligned}
$$

where $\rho$ is the bare conduction-electron density of states at $E_{F}$. Earlier [3] we have considered the exciton effects with neglecting spin fluctuations. We will see that the renormalization (4) results in new essential effects.

We have from Eqs.(6) and (7)

$$
\frac{G(C)}{G(0)}=\left[\frac{V(C)}{V(0)}\right]^{\alpha}, \alpha=-\frac{2 \lambda}{1+|\lambda|}
$$

so that $G(C)=G(0)$ in the incoherent regime. We derive from (6) in the incoherent and coherent cases, respectively

$$
\begin{aligned}
V(C) & =V(0)|D / w(C)|^{\rho G(0)} \\
V(C) & =V(0) /[1+G(0) \rho \ln |w(C) / D|]
\end{aligned}
$$

where $w(C)=C-E_{f}(C), D$ is a cutoff parameter of the order of bandwidth (we put in numerical calculations
$\left.D=\rho^{-1}=1\right)$. Then we have the closed equation for $w(C)$. In particular, for the incoherent regime

$$
\frac{\partial w(C)}{\partial C}=1+(N-1) \frac{\rho V^{2}(0)}{C}\left|\frac{D}{w(C)}\right|^{2 \rho G(0)}
$$

When $E_{f}$ lies sufficiently below the Fermi level (the Kondo regime), the quantity $|w(C)|$ can become small with decreasing $|C|$. We can use this condition to define the boundary between IV and Kondo cases. Formal definition of IV systems is the absence of solutions to the equation $w(C)=0$ which just determines the Kondo resonance (cf. Refs. 6, 17). Physically, Kondo lattice has a three-peak density of states (two Hubbard bands and the Kondo resonance), which is similar to the "doped Mott insulator" (note that in the dynamical mean-field theory (DMFT) the Hubbard model is reduced to the Anderson impurity model [15]). On the other hand, IV state is similar to the phase of strongly correlated metal: the Kondo peak as a separate solution is absent.

For $G(0)=0$, the boundary condition for the Kondo state is $\left|E_{f}(0)\right|>\Gamma=(N-1) \rho V^{2}(0)$. In the opposite IV case $|w(C)|$ remains finite. For $G(0) \neq 0, V(C)$ increases during the renormalization process, and the effective level width $\Gamma(C)$ becomes larger, so that the IV region becomes more wide. A temperature dependence of the energy gap (an increase with decreasing temperature) in IV compounds was observed experimentally in $\mathrm{SmB}_{6}$ [16], $\mathrm{YbB}_{12}$ and $\mathrm{Ce}_{4} \mathrm{BiPt}_{3}$ 2]. According to our treatment, the dependence of the effective hybridization $V(C)$ is non-monotonous: it passes through a maximum.

Now we consider in more detail the incoherent case which should be realized for diluted systems (the Anderson's localization prevents coherence at low temperatures). To present numerical results (Figs.1-4) we use the variable $\xi=\ln |D / C|$. As follows from (9), in the Kondo case the hybridization parameter $V(C)$ decreases practically by a jump when we approach the point $C=E_{f}(C)$, see Fig.2. With further decreasing $|C|$, a considerable region arises where we have to high accuracy $C \cong E_{f}(C)$ (Fig.1). More exactly, we have $\partial w(C) / \partial C \simeq 0$, so that we obtain from (11) near the maximum of $w(C)$

$$
w(C) \simeq-D\left|(N-1) \rho V^{2}(0) / C\right|^{1 / 2 \rho G(0)} .
$$

In this regime $V(C)=|C /[(N-1) \rho]|^{1 / 2}$, and the effective $s-f$ exchange parameter is $\rho I(C)=$ $\rho V^{2}(C) / E_{f}(C)=-1 /(N-1)=$ const.

In a standard consideration, the condition $C=E_{f}(C)$ determines an energy scale for a crossover to the regime of a heavy-fermion (Kondo) local Fermi liquid [13]. The "marginal" situation with $E_{f}(C) \cong C$ in a whole interval of the cutoff parameter $C$ means an essentially non-Fermi-liquid (NFL) picture. A similar mechanism of NFL behavior in magnetic Kondo lattices was proposed in Refs 7, 19 where a soft boson was obtained with the characteristic energy $\bar{\omega}(C) \cong|C|$. Note also that a 


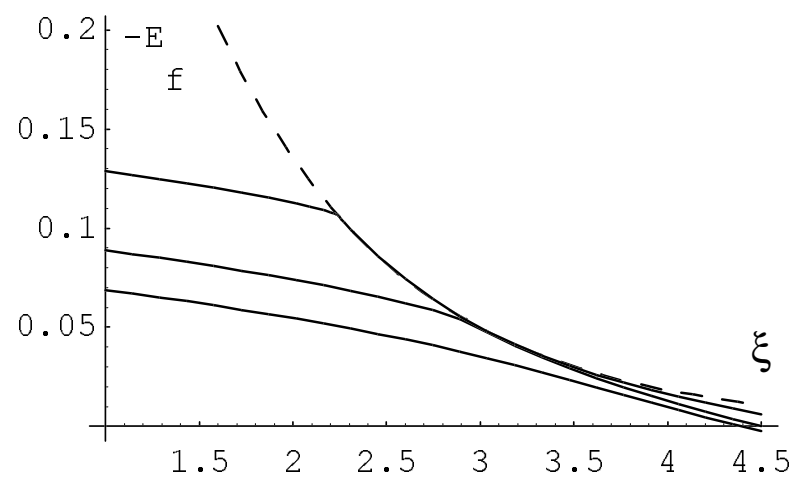

FIG. 1: Scaling trajectories $-E_{f}(\xi)$ for $V(0)=0.1, G(0)=$ $0.1, N=2$ in the incoherent case as compared to the curve $|C| / D=\exp (-\xi)$ (dashed line). The parameter values (for the curves from below to above) are $E_{f}(0)=$ $-0.08,-0.1,-0.14$.

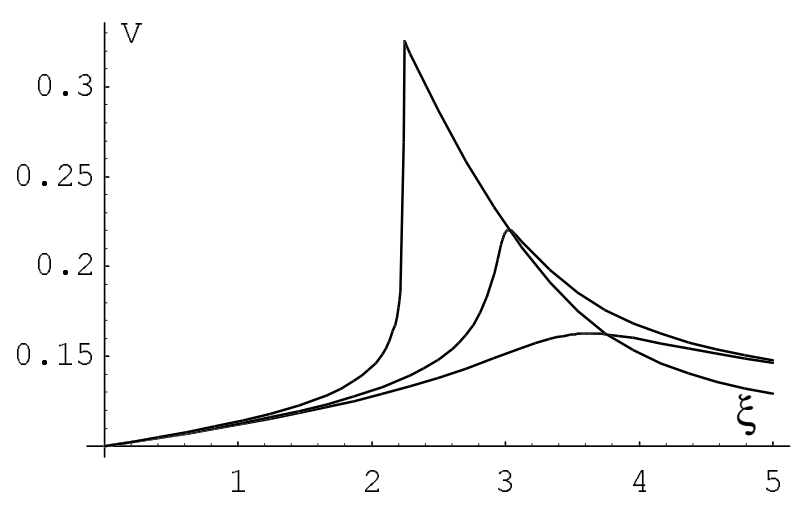

FIG. 2: Dependences $V(\xi)$ for the same parameter values as in Fig.1 (for the curves from below to above)

regime with the rate of the order parameter fluctuations $1 / \tau_{\phi}(T) \propto T$ is typical near a quantum phase transition [21]. Our situation is reminiscent of this regime in the sense that the characteristic valence fluctuation frequency $\max \left(\rho V^{2}(T),\left|E_{f}(T)\right|\right)$ is proportional to $T$ (after

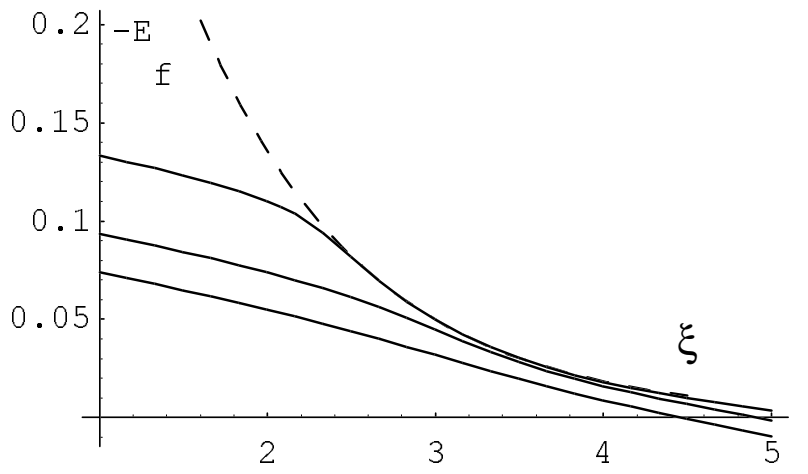

FIG. 3: Scaling trajectories $-E_{f}(\xi)$ for $V(0)=0.05, G(0)=$ $0.05, N=6, E_{f}(0)=-0.09,-0.11,-0.15$ in the coherent case.

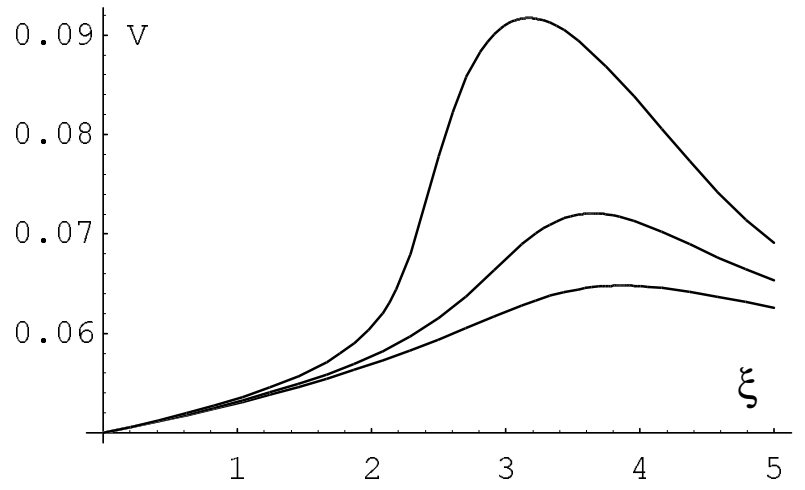

FIG. 4: Dependences $V(\xi)$ for the same parameter values as in Fig.3 (for the curves from below to above)

a natural replacement $|C| \rightarrow T)$.

Physically, the regime where a typical energy scale is just the temperature means a classical (MaxwellBoltzmann) electron gas (interacting with local momenta): heat capacity is approximately constant etc. Of course, in a pure form this behavior is never observed since, according to our results, it takes place only in a restricted temperature interval. However, we have a strong deviation from a simple scaling picture where we just enter strong-coupling regime, a characteristic "Kondo" temperature being $T$-independent. In particular, the Wilson number is nearly constant, but differs considerably from that in the singlet state $\left(I_{e f}=-\infty\right)$.

A similar "marginal" region in the dependence $E_{f}(C)$ occurs not only for the Kondo, but also for IV state near the critical line (in such a situation, the dependence $w(C)$ has a shallow minimum, and $V(C)$ a sharp maximum, see Figs.1-2).

To estimate the Kondo temperature we can use Haldane's arguments for the Anderson model with $N=2$ [13]. The generalization to arbitrary $N$ can be performed as (cf. Ref. 20)

$$
T_{K} \simeq D(\rho|I| N)^{1 / N} \exp (1 / \rho N I), I=V^{2} / E_{f}
$$

This expression is formally based on perturbation theory (two-loop scaling). However, Haldane noted that replacing in this formula both $D$ and $E_{f}$ by the characteristic energy scale $T^{*}=-C$, which is determined from the equation $C=E_{f}(C)$, yields the correct estimation for the Kondo temperature. As demonstrated above, in the presence of exciton effects (Falicov-Kimball interaction $G)$, a situation is possible where this equation holds approximately in a whole energy interval. The energy scale $T^{*}$ where the "marginal" regime starts is considerably changed by the exciton effects.

In the coherent case the last term in Eq.(5) results in a smearing of the singularity, especially for small $N$. However, with increasing $N$ the dependence $E_{f}(C)$ (Fig.3) becomes qualitatively similar to that in the incoherent case. On the other hand, the dependence $V(C)$ (Fig.4) is essentially modified even for $N \rightarrow \infty$. At $C \cong E_{f}(C)$ 
we have

$$
1=-(N-1) \rho V^{2}(C) / C-n[G(0) / V(0)] \partial V(C) / \partial C
$$

Solution to this Riccati equation is obtained in terms of the imaginary-argument Bessel and Macdonald functions $I_{p}(x)$ and $K_{p}\left(|(N-1) \rho C|^{1 / 2}\right)$ with $x=2 \mid(N-$ 1) $\left.\rho V^{2}(0) C\right|^{1 / 2} /[n G(0)], p=0,1$. For large $N$, the quantity $\partial V(C) / \partial C$ and consequently $V^{2}(\xi) / E_{f}(\xi)$ turn out to be practically $\xi$-linear near the maximum of $w(C)$. Thus we have a "classical" electron liquid with singular interactions which have logarithmic energy dependences. With further decreasing $|C|$ we have from Eq.(6)

$$
\partial V(C) / \partial C \propto|C|^{-1 / 2}=\left|E_{f}(C)\right|^{-1 / 2}
$$

so that the correction to $V^{2}(C) / E_{f}(C)$ is proportional to $|C|^{-1 / 2}$.

The $d-f$ Coulomb interaction can strongly renormalize the hybridization, which leads to the increase of the characteristic energy scale. In the incoherent regime it is a width of the resonance, in the coherent one the width of (indirect) gap or pseudogap [3]. The renormalization of the fluctuation rate $\rho V^{2}(C)$ can be very strong (about order of magnitude for realistic parameters). The corresponding temperature dependences can be found in both the regimes by the $\mathrm{RG}$ approach with the replacement $|C| \rightarrow T$.

In standard treatments of $\mathrm{HF}$ systems, one picks usually anomalous magnetic contributions to thermodynamic properties and compares them with exact results in the one-impurity Kondo problem. In particular, the dependences of the crystal-field level width from both ineleastic neutron scattering and nuclear magnetic resonance (NMR) have the form $\Gamma(T) \propto T^{1 / 2}$. In the Kondo resonance model such a behavior takes place above the Kondo temperature $T_{K}$, but in some cases $\left(\mathrm{CeB}_{6}, \mathrm{CePd}_{3} \mathrm{~B}_{0.6}, \mathrm{YbBe}_{13}\right)$ the dependence $T^{1 / 2}$ takes place at very low temperatures of a few K 22]. Further, the characteristic energy scale from linear heat capacity term $\gamma$ is of order of tens of Kelvins, whereas the temperature where $\gamma$ starts to deviate from constant is just a few of Kelvins. Thus there exists no unique energy scale. We have demonstrated that, indeed, the infrared behavior can be essentially different from the simple Anderson model owing to spin dynamics (see Refs 7, 19) and charge fluctuations (exciton effects) considered in the present work.

Of course, the estimations performed are qualitative since they are based on the continuation of perturbative Gellmann-Low scaling function to the strong coupling region. At the same time, the statement that exciton effects cannot be described by universal temperature independent $T_{K}$ seems to be reliable itself. Recently, direct ways of observing the Kondo resonance (STM) were proposed 23. As we know, they have been not yet applied to the IV systems. It would be interesting to compare the results for $T_{K}$ of these new experimental methods with those from investigating thermodynamic properties.

The research described was supported in part by Grant No. 747.2003.2 from the Russian Basic Research Foundation (Support of Scientific Schools).
[*] E-mail: Valentin.Irkhin@imp.uran.ru

[1] J. M. Lawrence, P. S. Riseborough, and R. D. Parks, Rep. Prog. Phys. 44, 1 (1981).

[2] P. S. Riseborough, Adv. Phys. 49, 257 (2000).

[3] V. Yu. Irkhin and M. I. Katsnelson, Sov. Phys. JETP 63, 631 (1986).

[4] Valence Instabilities and Related Narrow-Band Phenomena, ed. by R. D. Parks (Plenum Press, New York, 1977).

[5] Proc. Int. Conf. on Valence Fluctuations, J. Magn. Magn. Mater. $47 \& 48$ (1985).

[6] A. C. Hewson, The Kondo Problem to Heavy Fermions (Cambridge University Press, Cambridge, 1993).

[7] V. Yu. Irkhin and M. I. Katsnelson, Phys. Rev. B 56, 8109 (1997).

[8] R. Ramirez, L. M. Falicov, and J. C. Kimball, Phys. Rev. B 2, 3383 (1970); C. E. T. Goncalves da Silva and L. M. Falicov, J. Phys. C 5, 906 (1972).

[9] K. W. H. Stevens, J. Phys. C 9, 1417 (1976); J. Phys. C 11, 985 (1978).

[10] P. Schlottmann, Phys. Rev. B 22, 613 (1980); Phys. Rev. B 22, 622 (1980).

[11] D. L. Cox and A. Zawadowski, Adv. Phys. 47, 599 (1998).
[12] V. Yu. Irkhin and Yu. P. Irkhin, Sov. Phys. JETP 80, 334 (1995).

[13] F. D. M. Haldane, Phys. Rev. Lett. 40, 416 (1978).

[14] P. W. Anderson, J. Phys. C 3, 2346 (1970).

[15] A. Georges et al., Rev. Mod. Phys. 68, 13 (1996).

[16] T. S. Al'tshuler et al, JETP Lett. 40, 754 (1984).

[17] N. E. Bickers, Rev. Mod. Phys. 59, 845 (1987); O. Gunnarsson and K. Schönhammer, Phys. Rev. B 28, 4315 (1983).

[18] V. Yu. Irkhin and M. I. Katsnelson, Z. Phys. B 75, 67 (1989).

[19] V. Yu. Irkhin and M. I. Katsnelson, Phys. Rev. B 61, 14640 (2000).

[20] J. W. Rasul and A. C. Hewson, J. Phys. C 17, 2555 (1984).

[21] S. Sachdev, Quantum Phase Transitions (Cambridge University Press, Cambridge, 1999).

[22] P. Panissod, M.Benaki, and A. Qachaou, J. Phys.(Paris) 49, C8, 685 (1988).

[23] V. Madhavan et al, Science 280, 567 (1998). 